\title{
LAAPERTURA DE LA FRONTERA: ¿NUEVAS IDENTIDADES TRANSFRONTERIZAS?
}

\author{
Jan Mansvelt Beck \\ Universidad de Ámsterdam \\ jmansveltbeck@gmail.com \\ Luis Alfonso Hortelano Mínguez \\ Universidad de Salamanca \\ sito@usal.es
}

\section{RESUMEN}

Durante el apogeo de la soberanía se estudiaban las fronteras desde una perspectiva estado-céntrica. Hoy en día vivimos en una época de post-soberanía en que los estados ya no son contenedores de seguridad, economía y cultura y sus fronteras han perdido la función de barrera militar, económica y cultural. Por lo tanto nuevos enfoques teóricos se han desarrollado sobre las zonas fronterizas. Estos enfoques se basan en la idea de que la integración transnacional genera espacios de interacción económica, política y cultural, creando nuevas identidades transfronterizas. No obstante, los estudios de la frontera basados en estos nuevos enfoques padecen de una cierta euforia transfronteriza, porque hay indicios de que la apertura de la frontera no la deja como un palimpsesto de un pasado soberanista del estado. Por eso hay que matizar la euforia transfronteriza por las siguientes razones: primera, aunque se puede observar un incremento de la interacción económica transfronteriza, ésta sigue estando situada en su mayor parte dentro del estado; segunda, la cohesión político-cultural sigue siendo poco intensa en las zonas transfronterizas; y tercera, la frontera sigue dividiendo culturas e identidades.

Palabras clave: Teorías sobre zonas fronterizas, post-soberanía, identidades transfronterizas, fronteras abiertas.

Fecha de recepción: diciembre 2014.

Fecha de aceptación: septiembre 2015. 


\section{ABSTRACT}

During the heydays of sovereignty we have studied the borders from a state-centric perspective. Today we are living in an era of post sovereignty in which states are no more containers of security, economy and culture and borders have lost their role as military, economic and cultural barriers. As a result, new borderland theories have emerged. Innovative constructs are rooted in the idea that transnational integration generates spaces of economic, political and cultural interaction, creating new cross-border identities. However, borderland theories based on these new approaches suffer from cross-border euphoria because there are indications that the opening-up of the borders does not leave them as palimpsests of state sovereignty in the past. Therefore cross-border euphoria should be qualified for the following reasons: First, although economic cross-border interaction has increased, most interaction continues within the state. Second, politicocultural cohesion across the border has remained weak in borderlands. Third, the border continues dividing cultures and identities.

Keywords: Borderland theories, post sovereignty, cross-border identities, open borders.

\section{PRESENTACIÓN}

La globalización y la construcción de instituciones supranacionales han erosionado la relevancia de los estados-nación como «contenedores de poder» (Giddens 1985). Taylor (1994) ha señalado la pérdida del poder estatal, territorialmente sostenido a coste de poderosas redes urbanas cuyas influencias económicas sobrepasan las fronteras estatales. Además, los estados como centros de decisión sobre asuntos económicos se han debilitado por la emergencia de entidades como la Unión Europea (UE), la North American Free Trade Agreement (NAFTA) o la Association of Southeast Asian Nations (ASEAN) (O'Dowd 2001, Taylor 1994). Algo similar ha pasado con la autoridad de los estados en asuntos de seguridad, que hoy en día tienen que compartir con otros estados en organizaciones internacionales como la OTAN y las Naciones Unidas (Taylor \& Flint 2011). Estos procesos de globalización y transnacionalización han cambiado el rol de las fronteras estatales como barreras con una cierta porosidad hacia una apertura que, según unos autores implica una desterritorialización, que degrada las fronteras en fenómenos irrelevantes (Ohmae 1990, 1995). La apertura de las fronteras ha devaluado los enfoques estado-céntricos de las zonas transfronterizas y ha dado lugar a perspectivas enraizadas en la filosofía posmoderna. Estos enfoques posmodernos se basan en la idea de que la integración transnacional genera espacios de interacción económica, política y cultural, creando nuevas identidades transfronterizas. El objetivo de esta contribución es verificar las nuevas pretensiones teóricas mediante una confrontación empírica a base de unos estudios concretos de la actualidad transfronteriza. Para lograr este objetivo, se contestarán a las siguientes preguntas: primera, ¿en qué consisten básicamente las nuevas teorías sobre las fronteras y cuáles son las diferencias con las teorías desarrolladas en el apogeo de la soberanía estatal?; y segunda, ¿en qué medida los estudios empíricos recientes sostienen la hipótesis de que las fronteras abiertas crean nuevos espacios económicos, políticos, culturales e identitarios? 


\section{SOBERANÍA, POST-SOBERANÍA Y TEORÍA DE LA FRONTERA}

Cuando los estados eran soberanos se estudiaban las fronteras desde una perspectiva estado-céntrica. Durante el apogeo del estado-nación se podía considerar el estado como un «contenedor de poder» (Giddens, 1985). La soberanía del estado-nación implicaba respectivamente el ejercicio del poder exclusivo dentro del territorio, la separación de asuntos internos e internacionales y una delimitación de la sociedad, congruente con el territorio estatal (Agnew, 1997). ¿De qué ámbitos el estado es el contenedor de poder? Encontramos la respuesta en el artículo clásico del geógrafo británico Peter Taylor (1994). Taylor distingue cuatro ámbitos: bienestar social, seguridad, economía y cultura; de estos, los tres últimos tienen particular relevancia para las zonas fronterizas. De forma esquemática, la tabla 1 refleja el papel del estado según los ámbitos de dominio en las zonas fronterizas.

Tabla 1

SOBERANÍA: EL PAPEL DEL ESTADO EN LAS ZONAS FRONTERIZAS

\begin{tabular}{|l|l|l|}
\hline \multicolumn{1}{|c|}{ Dominio estatal } & \multicolumn{1}{c|}{ Zona fronteriza } & \multicolumn{1}{c|}{ Función de la frontera } \\
\hline Seguridad & Militarización & Barrera \\
\hline Economía & Aduanas & $\begin{array}{l}\text { Formal: barrera } \\
\text { Informal: interface }\end{array}$ \\
\hline Cultura e identidad & $\begin{array}{l}\text { Construcción nacional: aumenta } \\
\text { la discontinuidad }\end{array}$ & Barrera gana importancia \\
\hline
\end{tabular}

Fuente: Basado en Peter Taylor (1994).

El primer ámbito trata de la seguridad en términos de defensa contra amenazas exteriores. El papel del estado intentando garantizar la seguridad de su población se expresaba en la militarización de las zonas fronterizas, un proceso que ya había empezado antes de la época de soberanía y mucho antes de la emergencia de los estados-nación (Kolossov, 2006). Las infraestructuras militares construidas por el estado a lo largo de sus fronteras se han convertido en reliquias visuales del pasado que, en la actualidad, nadie duda de su valor patrimonial. En cuanto a la militarización fronteriza en Europa, aunque las fortificaciones construidas por el General e ingeniero francés Vauban desde finales del siglo XVII tienen mucha fama, Portugal ya tenía su precursor en la persona de Mateus Fernandes, que diseñó la fortificación de Almeida. En muchos países de la Europa contemporánea los artefactos militares han perdido su función, no solamente por falta de conflictos bélicos con estados vecinos, sino también porque las nuevas tecnologías militares han convertido en obsoletas las antiguas infraestructuras defensivas y, en definitiva, la frontera ha perdido su función de barrera militar (Figura 1).

El segundo ámbito en el que el estado ejercía un papel dominante era la economía. El estado intervenía en el mercado mediante impuestos, aranceles y proyectos para recabar ingresos. En el estado moderno los peajes internos desaparecían paulatinamente, mientras que, aparecían aduanas en las fronteras estatales. Formalmente la frontera funcionaba como muro para la interacción económica, salvo para los intercambios que se consideraban de utilidad para crear riqueza. Pero informalmente la frontera funcionaba como una «interface», 
Figura 1

FORTIFICACIONES EN LA FRONTERA HISPANO-LUSA

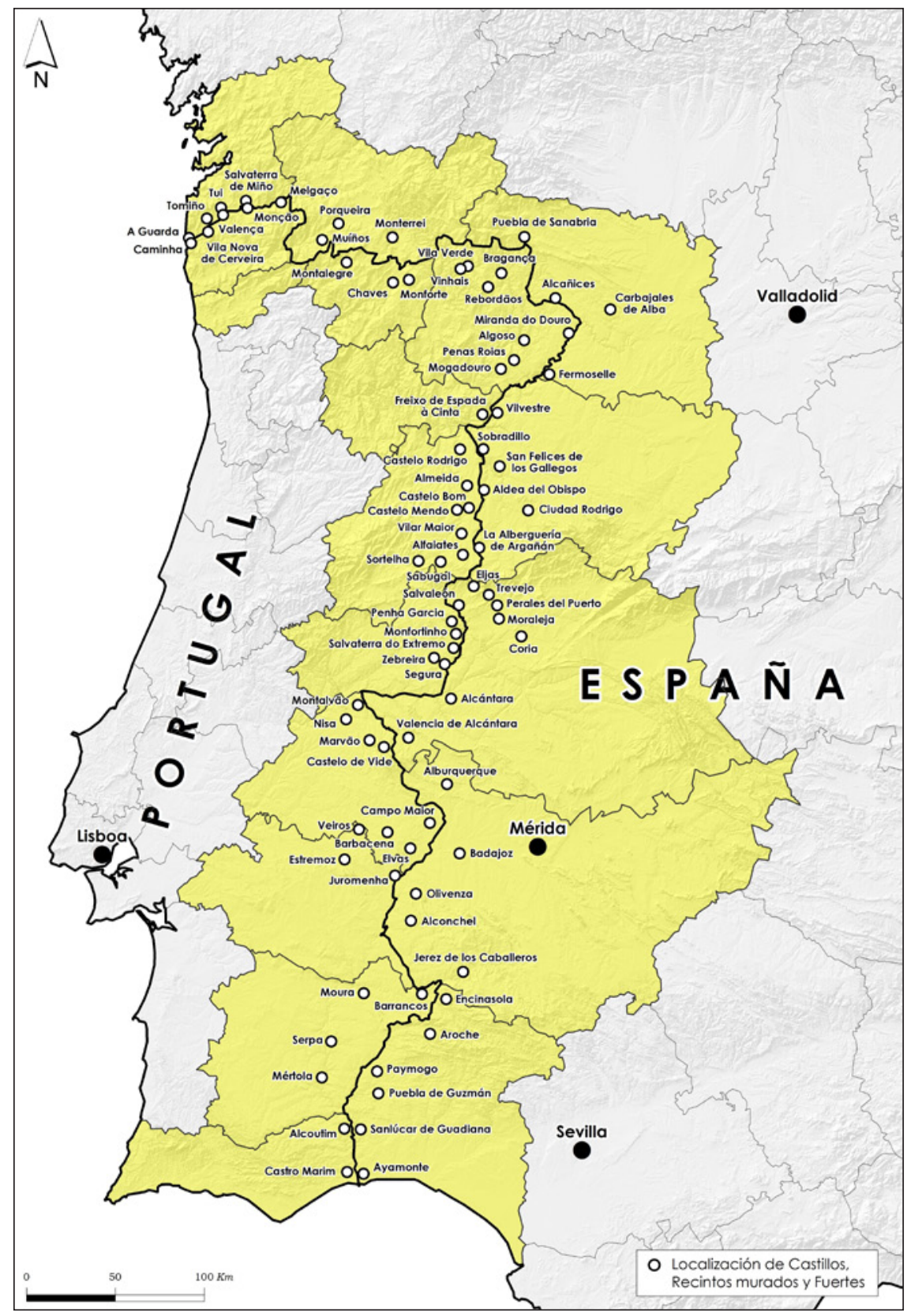

Fuente: Ministerio de Educación, Cultura y Deporte de España. Bienes Culturales Protegidos y Direção-Geral do Património Cultural de Portugal. Património cultural imóvel. 
dando lugar al contrabando de bienes y al turismo de compras. Con la creación de los mercados supranacionales y la correspondiente apertura de las fronteras la función de barrera económica ha perdido mucho peso (Taylor, 1994).

El tercer ámbito era el área cultural, ya que el estado tenía un dominio casi exclusivo en este área y, posteriormente, en la formación de las identidades nacionales. En la mayoría de los estados europeos el proceso de la nacionalización se manifestó a finales del siglo XIX o primeros del siglo XX, estando vinculado a la introducción de la educación obligatoria. La educación, los medios de comunicación y el servicio militar convirtieron campesinos en franceses (Weber, 1976) y pueblerinos en neerlandeses (Knippenberg \& De Pater, 2011). Las zonas fronterizas, que anteriormente se caracterizaban por una continuidad cultural en cuanto a lengua y costumbres y una identificación más bien local, empezaron a demostrar una discontinuidad cultural e identitaria demarcadas por la frontera. Incluso zonas pobladas por minorías culturales dentro del propio estado demostraban tal discontinuidad (Markusse, 2004). Por tanto, durante el apogeo del nacionalismo estatal, la frontera funcionaba cada vez más como barrera cultural e identitaria. En muchos países con minorías culturales el dominio estatal sobre la cultura se está debilitando por el auge de los nacionalismos periféricos con reivindicaciones culturales e identitarias. Mientras que el «contenedor del estado» en el campo de la seguridad y la economía está «goteando» por la globalización y la creación de poderosas entidades supranacionales, el de la cultura gotea por fuerzas dentro del propio estado (Taylor, 1994). Por supuesto, la función de las fronteras en la era de la post-soberanía es diferente del papel jugado durante el apogeo del soberano estado-nación (Tabla 2).

Tabla 2

POST-SOBERANÍA: EL PAPEL DE LA FRONTERA SEGÚN DOMINIO

\begin{tabular}{|l|l|l|}
\hline \multicolumn{1}{|c|}{ Dominio } & \multicolumn{1}{c|}{ Nivel } & \multicolumn{1}{c|}{ Frontera } \\
\hline Seguridad & Supra-estatal & Barrera o interface \\
\hline Economía & Supra-estatal y global & Interface o irrelevante \\
\hline Cultura & $\begin{array}{l}\text { Sub-estatal y estatal en estados multiculturales } \\
\text { Estatal en estados monoculturales }\end{array}$ & $\begin{array}{l}\text { Interface para interacción } \\
\text { Intracultural o intercultural }\end{array}$ \\
\hline
\end{tabular}

Fuente: elaboración propia.

Hoy en día vivimos en una época de post-soberanía en el que los estados ya no son contenedores de seguridad, economía y cultura y sus fronteras han perdido la función de barrera militar, económica y cultural. Lógicamente nuevos enfoques teóricos se han desarrollado sobre las zonas fronterizas. Estos enfoques se basan en la idea de que la integración transnacional genera espacios de interacción económica, política y cultural, creando nuevas identidades transfronterizas. Uno de los geógrafos que más ha trabajado sobre la integración transfronteriza en Europa es el escocés Michael Keating. Keating (1998) insinúa un nuevo regionalismo transfronterizo, que no está contenido por el estado, considerando las alianzas de zonas transfronterizas como manifestaciones del regionalismo extra-estatal y regiones en construcción. En los apartados siguientes se analiza hasta qué punto se trata de la formación de regiones transfronterizas, respectivamente en el sentido económico, político, cultural e identitario. 


\section{II.1. Zonas fronterizas, apertura e interacción económica}

Con la integración europea se puede observar un incremento de la interacción económica transfronteriza. Pero hay tres indicios de que el aumento del comercio internacional no ha contribuido mucho a la formación de «Euroregiones».

El primer indicio es de carácter general. Yeung (1998) criticando la visión casi profética de Ohmae $(1990,1995)$ de un mundo sin fronteras, observa que cada estado sigue teniendo su propio sistema de regulaciones y, por tanto, las condiciones para desarrollar actividades económicas varían según el estado.

El segundo indicio muestra que la interacción económica sigue estando situada en su mayor parte dentro del estado. Por ejemplo, los análisis a base de tablas imput-output para la Comunidad Autónoma del País Vasco demuestran que el tejido vasco intercambia entre 12 y 16 veces más con el resto de España que con cualquier otro país europeo. Curiosamente el discurso nacionalista vasco presenta al País Vasco como una región transfronteriza muy integrada y que, también, esta idea es usada en publicaciones científicamente dudosas (véase por ejemplo Gómez Piñeiro, 1985). Otros estudios presentan resultados parecidos, como el de MacCallum (1995) en el caso de la frontera abierta entre los Estados Unidos y Canadá e igual que el estudio de Van Houtum (1997) sobre la zona fronteriza entre los Países Bajos y Alemania.

El tercer indicio, como señala Clement (1997: 55-56) en su reseña bibliográfica de la literatura sobre la economía de las zonas fronterizas, es que la desaparición de las fronteras no asegura el desarrollo para estas zonas. Incluso, el desarrollo podría ser más difícil, porque la nueva configuración será más competitiva. Por eso muchas zonas fronterizas se quedarán en la periferia, tanto geográfica como económica. Basta decir que la mayoría de las regiones fronterizas son periferias en sus países y en sus regiones (Rumley \& Minghi, 1991). En el caso de Portugal y España hay variables de que las relaciones entre los centros económicos pueden reforzar su posición económica dentro de los respectivos estados. Sánchez Hernández (1998) demuestra que la interacción entre Portugal y España por la vía Irún-Aveiro refuerza más las zonas industrializadas a ambos extremos del eje que en la cercanía de la frontera. Aunque el turismo fronterizo entre España y Portugal ha experimentado un tímido crecimiento, no ha podido frenar el declive demográfico que se manifiesta en un acusado envejecimiento, en una débil densidad poblacional y en una progresiva despoblación (Sánchez López \& Cabero Diéguez, 1994: 71). Así, la Raya ibérica («a raia») y las otras zonas fronterizas continúan siendo periferias económicas (Campesino Fernández 2013, 2014; Campesino Fernández \& Jurado Almonte, 2014). A pesar de que múltiples autores (Perkmann, 2003 y Scott, 2000) enfatizan el éxito de la construcción de instituciones transfronterizas en Europa, justamente en las más antiguas Euroregiones, es decir en la frontera Holando-Alemana, están entrando dudas sobre el éxito económico e institucional de la integración fronteriza (Jacobs \& Kooij, 2013). Por ejemplo, las diferencias en cuanto al PIB entre los territorios administrativos NUTS III de ambos lados de la frontera no avanzan hacia la convergencia y demuestran una cierta discontinuidad (Figura 2). Estas dudas las comparten un número creciente de estudiosos de la integración transfronteriza en Europa (para una reseña, véase Van Houtum, 2000: 66). 


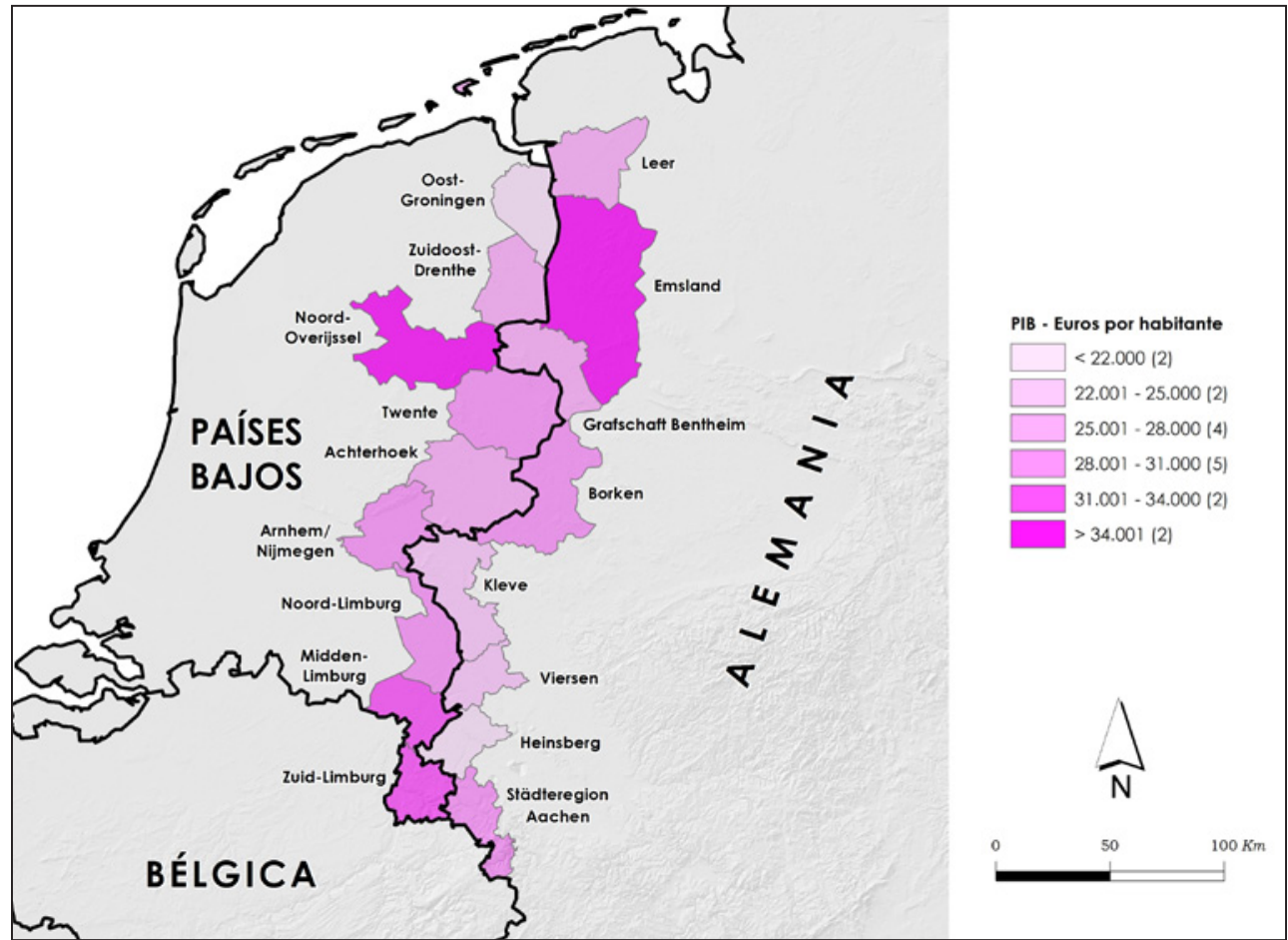

Fuente: Oficina Europea de Estadística (EUROSTAT). General and regional statistics. 2009.

\section{II.2. Zonas fronterizas, apertura y cohesión político-institucional}

Después de la apertura de las fronteras europeas Rifkin (2004) preveía un futuro de una Europa unida más prometedor que el de los Estados Unidos. Este espíritu también existía cuando la UE, los estados miembros de la UE, gobiernos regionales y autoridades locales empezaron a estimular la cooperación transfronteriza ${ }^{1}$. Efectivamente, en muchos casos los europeos han sido capaces de construir instituciones transfronterizas para sus proyectos transfronterizos. Pero la cohesión política e institucional ha sido de baja intensidad, a pesar de historias de éxito como en las más antiguas Euroregiones; es decir, los proyectos transfronterizos entre los Países Bajos y, Alemania y Bélgica respectivamente. A pesar de la creación de estas instituciones el desencanto está creciendo. Una de las causas principales

1 Para facilitar la cooperación territorial en el marco comunitario, tanto a nivel estatal, como a nivel local y regional, fue aprobado el Reglamento 1082/2006 del Parlamento Europeo y del Consejo, de 5 de julio de 2006, sobre la Agrupación Europea de Cooperación Territorial (AECT). Esta norma ha creado una nueva figura, las Agrupaciones Europeas de Cooperación Territorial (AECT), que permite a los Estados miembros, a los entes locales y regionales, y a sus organismos dependientes, crear un nuevo tipo de entidad territorial dotada de personalidad jurídica con el fin de reforzar la cohesión económica y social en el ámbito de la Unión Europea. 
de esta desilusión es la persistencia de la frontera estatal, que sigue dividiendo los sistemas legislativos. Por tanto, a cada lado de la frontera siguen existiendo distintos conjuntos de regulaciones que impiden una integración fructuosa (De Vries 2008a, 2008b; Jacobs \& Kooij 2013, Knippenberg 2004). En el caso de la cooperación transfronteriza entre Flandes y Holanda, la falta de integración política es llamativa, porque la lengua, el neerlandés, se habla a ambos lados de la frontera. Eso no es un hecho aislado en otras zonas que comparten el mismo idioma, como Austria-Tirol del Sur, Alemania, la parte germanófona de Bélgica, o las zonas euskaldunes de España y Francia (Markusse 2004, Mansvelt Beck 1999, 2008, 2011). En estos casos no es el idioma que sigue separando a la población, sino sobre todo, lo que Brunet-Jailly (2005) llama la ausencia de la «political clout», el peso político común, que teóricamente puede unir grupos humanos que dentro de sus respectivos estados forman minorías étnicas. En el caso vasco, incluso las fronteras intra estatales, es decir entre Navarra y Euskadi impiden la integración política e institucional (Mansvelt Beck 2008). Además Bray (2004) ha observado la existencia de diferentes culturas políticas al norte y al sur de la frontera estatal.

El caso de la frontera entre Irlanda del Norte e Irlanda parece único. Anderson (2002) observa la emergencia de una democracia transfronteriza acompañada de la construcción de instituciones comunes. No es como en otros casos, una cooperación política iniciada por elites nacionales y locales, sino un movimiento que ha nacido desde abajo y, que finalmente, ha ayudado a la pacificación de Irlanda del Norte. Este caso es la excepción de la regla. Como veremos en el apartado siguiente los movimientos etno-nacionalistas de carácter transfronterizo, como el caso vasco, generalmente carecen de peso político.

Finalmente, la existencia de instituciones transfronterizas en sí no dice mucho, porque suele ser la obra de élites políticas. Por ejemplo, el caso de cooperación entre Saar (Alemania), Lorraine (Francia) y Luxemburgo está representado como un éxito de colaboración entre municipios y administraciones estatales y sub-estatales (Schulz, 1999). Schulz llama a la creación de instituciones comunes «desarrollo desde abajo», pero no nos informa sobre una posible identificación de la población con el nuevo espacio. Las instituciones pueden formar nuevos espacios, pero generalmente estos quedan aislados de la sociedad local, porque la población carece de una conciencia compartida de pertenecer a una región (Paasi, 2001). Esta conciencia no es un fenómeno muy extendido, como demuestra el resultado de una encuesta en las Euroregiones entre los Países Bajos y Alemania donde el 70\% de los habitantes no sabía que vivía en una región transfronteriza dotada de instituciones singulares (Reichenbach et al., 1999). Tomando en consideración la falta de una conciencia colectiva después de decenios de proyectos transfronterizos, es muy probable que la emergencia de las nuevas identidades espaciales no esté relacionada con la duración de la cooperación.

\section{II.3. Zonas fronterizas, apertura, innovación cultural e identitaria}

Los habitantes de las zonas fronterizas comparten historias de nacionalización desde el centro cultural del estado que, en muchas ocasiones, también suele ser el centro político. La nacionalización consiste en la homogeneización administrativa y cultural a costa de idiomas regionales, costumbres locales (Weber 1976, Gellner 1983) y una creciente 
identificación con el estado-nación. Anderson (1991) llama la identificación con la nación «la comunidad imaginada», que según él tiene sus orígenes en el capitalismo de imprenta. Tilly (1992) ve la nacionalización como una consecuencia de las guerras y la introducción del servicio militar, mientras que Deutsch (1966) enfatiza la creciente comunicación social por medios de comunicación, migraciones internas e integración económica que alargaban el horizonte mental desde la localidad hasta el estado-nación. En los países desarrollados el estado producía cada vez más bienes colectivos, como infraestructuras, educación, sanidad y otros servicios públicos. «El conjunto de estos bienes puede dar legitimidad al estado, lo que Mann (1986) llama el poder infraestructural del estado». Al final del proceso de nacionalización el sentido de pertenecer a una nación se vuelve tan normal que Billig (1995) lo llama nacionalismo banal. Hay casos en que los procesos de nacionalización de las poblaciones fronterizas han sido exitosos, mientras que, en otras ocasiones han fracasado (Knippenberg \& Markusse, 1999).

Si se trata de una nacionalización completa de una zona fronteriza tanto la cultura como la identificación son completas. Culturalmente los habitantes son ciudadanos del estado, hablando el idioma oficial, mientras lo que queda de las costumbres locales frecuentemente sufre de folklorización. La identificación es con el estado, aunque si existen múltiples identidades espaciales serán con regiones o localidades dentro del mismo estado.

Tabla 3

NACIONALIZACIÓN DE ZONAS FRONTERIZAS Y PROBABILIDAD DE INTERACCIÓN CULTURAL Y FORMACIÓN DE IDENTIDADES ESPACIALES TRANSFRONTERIZAS

\begin{tabular}{|c|c|c|c|}
\hline \multicolumn{2}{|c|}{ Nacionalización } & \multirow[b]{2}{*}{ Culturas } & \multirow{2}{*}{$\begin{array}{l}\text { Probabilidad de interacción } \\
\text { cultural y formación de } \\
\text { identidades transfronterizas }\end{array}$} \\
\hline Lado 1 (L1) & Lado2 (L2) & & \\
\hline \multirow{2}{*}{ Completa } & \multirow{2}{*}{ Completa } & Distintas & Muy baja \\
\hline & & Iguales & Baja \\
\hline \multirow[b]{2}{*}{ Completa } & \multirow[b]{2}{*}{ Incompleta } & Distintas & Muy baja \\
\hline & & $\begin{array}{l}\text { Cultura L1 = Cultura } \\
\text { minoritaria en L2 }\end{array}$ & $\begin{array}{l}\text { Baja, pero aumenta con el peso de } \\
\text { etnoregionalismo L2 }\end{array}$ \\
\hline \multirow[b]{2}{*}{ Incompleta } & \multirow[b]{2}{*}{ Incompleta } & Distintas & Muy baja \\
\hline & & $\begin{array}{l}\text { L1 y L2 comparten culturas } \\
\text { minoritarias }\end{array}$ & $\begin{array}{l}\text { Aumenta con el peso de } \\
\text { etnoregionalismo en L1 y L2 }\end{array}$ \\
\hline
\end{tabular}

La tabla 3 demuestra que solo cuando a un lado de la frontera hay una cultura minoritaria que tiene las mismas características que la cultura estatal dominante al otro lado de la frontera, hay alguna probabilidad de que se produzca una identidad transfronteriza, sobre todo cuando hay un fuerte etnoregionalismo en la población minoritaria. No obstante, la identificación tendrá un carácter asimétrico, ya que la población nacionalizada se sigue identificando con su propio estado. Culturalmente la homogenización de minorías culturales no toma la forma de una asimilación total. En este caso la hibridación cultural ocurrirá, frecuentemente caracterizada por el bilingüismo o el uso de la lengua oficial junto con el 
dialecto. Pero incluso si a ambos lados de la frontera viven minorías culturales que se identifican como distintas de la mayoría de la población no es una condición suficiente para formar una nueva etnoregión.

Dos ejemplos, respectivamente el de Holanda y Flandes y el vasco, demuestran que semejanzas culturales a ambos lados de la frontera no cristalizan automáticamente en identidades transfronterizas. En los Países Bajos la población en la frontera belga está completamente nacionalizada. Suelen hablar el néerlandes, junto con el dialecto local y se identifican como neerlandeses. Los flamencos del otro lado se sienten más flamencos que belgas, pero no se identifican con los Países Bajos, a pesar de hablar el mismo idioma. Incluso el único lazo cultural que une Flandes y los Países Bajos, la Nederlandse Taalunie (la unión lingüística del idioma neerlandés) corre peligro por conflictos sobre la gramática, la ortografía y la gestión (De Volkskrant, 2015).

El segundo ejemplo seleccionado es el País Vasco (Mansvelt Beck, 2008). Los vascos de Euskadi se ven de forma mayoritaria como vascos (es decir únicamente vascos o más vascos que españoles), mientras que en el país vasco francés la mitad se ve como vascos y la otra fracción como franceses. En Navarra, donde vive también una población con rasgos culturales vascos, predomina la identidad regional navarra. En todo el conjunto hay una cierta hibridación. El discurso nacionalista vasco identifica Euskal Herria como el país de los vascos, incluyendo todos los territorios mencionados. En Euskadi, de cultura menos euskaldún que el país vasco francés, donde los nacionalistas vascos siempre han dirigido la Comunidad Autónoma, los habitantes se identifican con Euskal Herria. En Euskadi se ha producido una banalización de Euskal Herria, que se refleja en los mapas meteorológicos en los dos periódicos regionales más leídos, El Correo y El Diario Vasco, ambos de tendencia no nacionalista. Los vascos de Francia, a pesar de ser culturalmente bastante más vascos, no han traducido su estado de minoría cultural en reivindicaciones etnonacionalistas, lo que demuestran los resultados de las elecciones cantonales en que los partidos estatales siempre han obtenido grandes mayorías. En el periódico regional más leído, Le Sud Ouest, los mapas meteorológicos exponen Aquitania sin ninguna referencia a las zonas vascas al sur de la frontera. La falta de peso político etnonacionalista y de banalización del nacionalismo vasco, también destacan en Navarra. En el caso vasco se puede concluir que, sí existe una identidad transfronteriza, pero la identificación con el nuevo espacio es asimétrica, ya que está escasamente compartida fuera de la Comunidad Autónoma del País Vasco. Recientemente Keating, el protagonista del nuevo enfoque, reconoce en un estudio empírico que la construcción de un territorio vasco transfronterizo no se ha podido realizar (Bray \& Keating, 2013).

El caso del País Vasco se enmarca en el contexto de la frontera hispano-francesa, donde encontramos otros ejemplos, como el concerniente a los Països Catalans o Països de Llengua Catalana. La herencia histórica y el peso demográfico juegan un papel significativo en el mantenimiento de la identidad a ambos lados de la muga; mientras que, en la vertiente francesa se ha producido una asimilación estatal, en la margen española persisten los rasgos identitarios etnoregionales. Las diferencias de identidad en la franja transfronteriza hispano-francesa también se manifiestan en las disimetrías demográficas, en cuanto al grado de ocupación del territorio, y administrativas en lo relativo a la lejanía geográfica y cultural de las capitales regionales, principalmente, de las sedes francesas (Figura 3). 


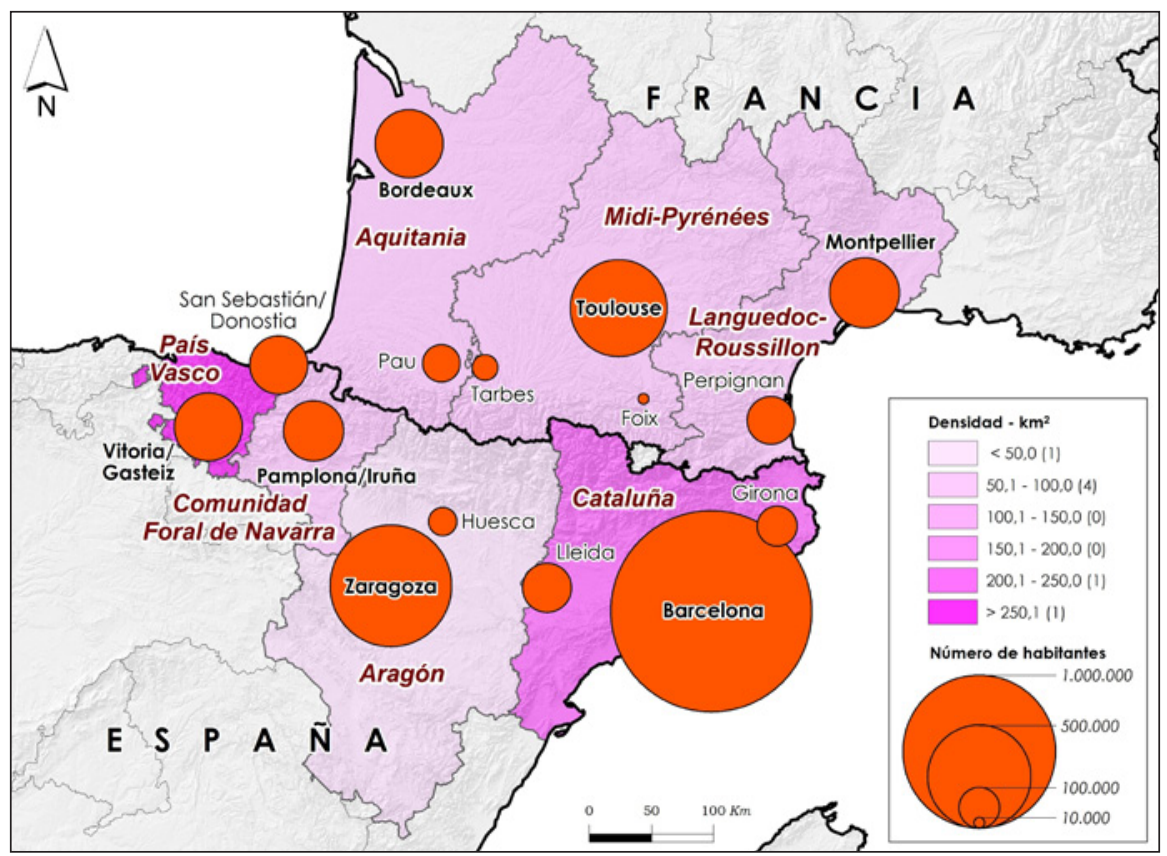

Fuente: Oficina Europea de Estadística (EUROSTAT). General and regional statistics. 2015.

\section{A MODO DE CONCLUSIÓN: MATIZACIONES A LA EUFORIA TRANSFRONTERIZA}

El final de los estados soberanos ha llevado al abandono de teorías que perciben las fronteras como barreras que separan entidades territoriales. El hecho de que la permeabilidad de las fronteras en la época post soberana haya aumentado, ha generado nuevos tipos de interacción político-administrativa, económica y cultural en Europa estimulados por la Unión Europea (UE). Ante el fenómeno de la aparición de nuevos lazos transfronterizos han surgido modernas teorías sobre las fronteras, que interpretan las zonas transfronterizas como regiones emergentes con su propia economía y una personalidad político-cultural, acompañados de modernas identidades espaciales.

No obstante, se ha demostrado que los estudios de la frontera basados en estos noveles enfoques padecen de una cierta euforia transfronteriza, porque hay indicios de que la apertura de la frontera no la deja como un palimpsesto de un pasado soberanista del estado. Los estudios empíricos sobre la apertura de las fronteras europeas demuestran que la interacción transfronteriza existe, pero no deja de ser un fenómeno insignificante. La construcción de entidades transfronterizas generalmente consiste en instituciones poco enraizadas en la sociedad y solo involucra cosméticamente a los ciudadanos. El aumento de la interacción económica apenas deja huellas en el desarrollo regional porque la frontera continúa como una periferia económica y no ha disminuido la brecha provocada por las desventajas de localización en relación al resto 
de territorios. La cohesión política y cultural no prospera, porque más de un siglo de nacionalización de las zonas fronterizas sigue separando administraciones, entidades territoriales, sistemas de regulación, culturas y, sobre todo, identidades espaciales.

La gran mayoría de estudios empíricos nos informan sobre la cooperación político-administrativa y las actividades económicas, pero proporcionan poquísimos datos sobre la integración cultural. Además, carecen de estudios sobre identidades transfronterizas emergentes. No obstante, los pocos indicios que hay, demuestran que las nuevas identidades fronterizas, postuladas en enfoques posmodernos, son visiones de un futuro que todavía no se ha materializado.

\section{BIBLIOGRAFÍA}

ANDERSON, B. (1991): Imagined Communities, Reflections on the Origin and Spread of Nationalism. London. Verso.

ANDERSON, J. y D. HAMILTON (2002): «Transnational democracy versus national conflict: border crossings in Ireland», en J. Anderson (Dir.), Transnational Democracy, Political spaces and border crossings. London/New York, Routledge, 129-148.

BILLIG, M. (1995): Banal Nationalism. London. Sage.

BRAY, Z. (2004): Living Boundaries. Frontiers and Identity in the Basque Country. Bruselas, Presses Interuniveritaires Europeénnes.

BRAY, Z. y KEATING, M. (2013): «European integration and the Basque Country in France and Spain», en Mabry, T. J., J. McGarry, M. Moore, \& B. O’Leary (Dirs.) Divided nations and European integration. Philadelphia: University of Pennsylvania Press, 127-156.

BRUNET-JAILLY, E. (2005): «Theorizing borders: an interdisciplinary perspective». Geopolitics, 10, 633-49.

CAMPESINO FERNÁNDEZ, A.J. (Dir.) (2013): Turismo de frontera (I). Oferta y demanda turística en la Raya ibérica. Vigo. Rede Ibérica de Entidades Transfrontereiças.

CAMPESINO FERNÁNDEZ, A.J. (Dir.) (2014): Turismo de frontera (II). Oferta y demanda turística en la Raya ibérica. Cáceres. CopeGraf, s.l.

CAMPESINO FERNÁNDEZ, A. y JURADO ALMONTE, J.M. (Dirs.) (2014): Turismo de frontera (III). Productos turísticos en la Raya ibérica. Huelva. Universidad de Huelva.

CLEMENT, N.C. (1997): «The Changing Economics of International Borders and Border Regions», in P. Ganster, A. Sweedler, J. Scott and W.-D. Eberwein (Eds.) Borders and Border Regions in Europe and North America. San Diego, Cal.: San Diego State University Press, 47-63.

DEUTSCH, K. (1966): Nationalism and Social Communication. 2nd edn. New York: MIT.

DE VOLKSKRANT (2015): Taalunie dreigt uiteen te vallen. http://www.volkskrant.nl/wetenschap/taalunie-dreigt-uiteen-te-vallen a4064308/ (visitado 25-07-2015), 9-07-2015

DE VRIES, J. (2008a): «Breaking the Deadlock: Lessons from Cross-Border Spatial Projects in Flanders and the Netherlands». DISP The Planning Review, 44, 48-61.

DE VRIES, J. (2008b): «Cross-border co-operation in the Rhine-Scheldt Delta: The long road of institution building», in M. Leibenath, E. Korcelli-Olejniczak \& R. Knippschild (Dir.) Cross-border Governance and Sustainable Spatial Development, Mind the Gaps! Berlin, Springer, 51-66. 
GELLNER, E. (1983): Nations and Nationalism. Oxford. Basil Blackwell.

GIDDENS, A. (1985): The nation-state and violence. Cambridge. Polity Press.

GIL-PAREJA, S.; LLORCA-VIVERO R. y MARTÍNEZ-SERRANO J.A. (2006): «The border-effect in Spain: the Basque Country case». Regional Studies 40, 335-345.

GÓMEZ PIÑEIRO, F. J. (1985): Geografía de Euskal Herria. Barcelona. Oikos-Tau.

HORTELANO MÍNGUEZ, L. A. (2014): «Dinámicas actuales en la "raya" de Castilla y León y Portugal: despoblación, atonía económica y desvertrebración interna», en Rui Jacinto (Coord.): Espaços de Fronteira, Territorios de Esperanza. Das Vulnerabilidades às Dinâmicas de Desenvolvimento. Iberografías n ${ }^{\circ} 27$. Guarda, Centro de Estudios Ibéricos (CEI), 131-139.

JACOBS, J. y KOOIJ, H.J. (2013): «Fading EUphoria at the Dutch-German Border? The Case of Avantis». Tijdschrift voor economische en sociale geografie, 104, 379-387.

KEATING, M. 1998): The New Regionalism in Western Europe: Territorial Restructuring and PoliticalChange. Cheltenham. Edward Elgar.

KOLOSSOV, W. (2006): «Theoretical limology: postmodern analytical approaches».Diogenes, 210, 5, 11-22.

KNIPPENBERG, H. y B. de PATER. (1988): De eenwording van Nederland, Schaalvergroting en integratie sinds 1800. Nimega. SUN.

KNIPPENBERG, H. y MARKUSSE, J. (Dirs.) (1999): Nationalising and Denationalising European Border Regions, 1800-2000, Views from Geography and History. Dordrecht. Geojournal Library Series. Kluwer.

KNIPPENBERG, H. (2004): «The Maas-Rhine Euroregion: A Laboratory for European Integration?». Geopolitics, 9, 608-626.

LÓPEZ TRIGAL, L. y GUICHARD, F. (Coords.) (2000). La frontera hispano-portuguesa: Nuevo espacio de atracción y cooperación. Salamanca. Fundación Rei Afonso Henriques.

MANN, M. (1986): The autonomous power of the state: its origins, mechanisms and results, en Hall, J.A. (Dir.), States in History. Oxford. Basil Blackwell.

McCALLUM, J. (1995): «National Borders Matter: Canada-U.S. Regional Trade Patterns». The American Economic Review, 85, 3, 615-623.

MANSVELT BECK, J. (1999): «An Anatomy of Basque Identity, a Geographical Analysis of Identity Patterns in the Franco-Spanish Border Area», en H. Knippenberg \& J. D. Markusse (Dirs.), Nationalising and Denationalising European Border Regions, 18002000, Views from Geography and History, Dordrecht: Geojournal Library Series. Kluwer, 153-172.

MANSVELT BECK, J. (2008): «Has the Basque borderland become more Basque after opening the Franco-Spanish border?». National Identities, 10, 4, 373-388.

MANSVELT BECK, J. (2011): Post-Sovereignty: Reframing the Basque Borderland?, en Jolán Róka (Dir.) Globalisation, Europeanisation and Other Transnational Phenomena. Budapest. Budapesti Kommunikációs és Üzleti Fóskola, 238-252.

MARKUSSE, J. (2004): «Transborder regional alliances in Europe: changes for ethnic Euroregions?». Geopolitics, 9, 4, 649-673.

O'DOWD, L. (2001): «Analysing Europe's borders». IBRU Boundary and Security Bulletin, 67-79.

OHMAE, K. (1990): The borderless world: power and strategy in the interlinked economy. Harper Collins. London 
OHMAE, K. (1995): The end of the nation state: the rise of regional economies. Harper Collins. London.

PAASI, A. (2001): «Europe as a Social Process and Discourse: Considerations of Place, Boundaries and Identity». European Urban and Regional Studies 8, 1, 7-28.

PERKMANN, M. (2003): «Cross-border regions in Europe. Significance and drivers of regional cross-border cooperation». European Urban and Regional Studies, 10, 2, 153 171.

REICHENBACH, E.; SPOORMANS, H. y KORSTEN, A. (1999): Grensoverschrijdende samenwerking, en H. Spoormans, E. Reichenbach and A. Korsten (Dirs.), Grenzen Over:Aspecten van grensoverschreidende samenwerking. Bussum. Coutinho.

RIFKIN, J. (2004): The European dream: how Europe's vision of the future is quietly eclipsing the American dream. Cambridge. UK, Polity.

RUMLEY, D. y MINGHI, J.V. (1991): Introduction: the border landscape concept, en D. Rumley \& J. V. Minghi (Dirs.), The geography of border landscapes. London/New York, Routledge.

SÁNCHEZ HERNÁNDEZ, J. L. (1998): El eje Irún-Aveiro. Geografía de un eje de desarrollo. Salamanca. Caja Duero.

SÁNCHEZ LÓPEZ, F. y CABERO DIÉGUEZ, V. (1994): La frontera hispano-portuguesa en el marco de la nueva Europa: la region fronteriza de Salamanca. Salamanca. Instituto de Recursos Naturales y Agrobiología y Departamento de Geografía de la Universidad de Salamanca.

SCHULZ, C. (1999): City-naetworking and bottom-up development: the influence of local authorities in the Saar-Lor-Lox Region, en H. Knippenberg \& J. Markusse (Dirs.) (1999) Nationalising and Denationalising European Border Regions, 1800-2000, Views from Geography and History, Dordrecht: Geojournal Library Series, Kluwer, 223-240.

SCOTT, J. (2000): «Euroregions, Governance and Transborder Co-operation within the EU». European Research in Regional Science, 10, 104-15.

TAYLOR, P. (1994): «The state as container: territoriality in the modern word-system». Progress in Human Geography, 18, 151-162.

TAYLOR, P.J. y FLINT, C. (2011): Political Geography: World-Economy, Nation-State and Locality. New Jersey. NJ, Prentice Hall.

TILLY, C. (1992): Coercion, Capital and European States AD 990-1992. Cambridge. MA/ Oxford, UK: Blackwell.

VAN HOUTUM, H. (1997): The Development of Cross-border Economic Relations. Tilburg. Center for Economic Research. Tilburg University.

VAN HOUTUM, H. (2000): «An overview of European geographical research on borders and border regions». Journal of Borderland Studies, 15, 1, 57-83.

WEBER, E. (1976): Peasants into Frenchmen, The Modernization of Rural France, 18701914. Stanford. Stanford University Press.

YEUNG, H. (1998): «Capital, State and Space: Contesting the Borderless World». Transactions of the Institute of British Geographers, 23, 3, 291-309. 